\title{
Computer Simulation of Sputter-Deposition Processes
}

\section{Eisuke SUZUKI and Yoichi HOSHI}

\author{
Faculty of Engineering, Tokyo Institute of Polytechnics, Atsugi, Kanagawa 243-02, Japan
}

\begin{abstract}
Sputter deposition process was investigated by computer simulation in order to clarify the effects of sputter-deposition parameters (sputtering gas pressure, substrate temperature, and high-energy particle bombardment of the film surface) on the microstructure of the film. Changes in sputtering gas pressure lead not only to changes in the distributions of the incidence angles and energy distribution of deposition particles, but also to changes in the film composition. The gas pressure dependence of the film composition in magnetron sputtering differs significantly from that in facing target sputtering. The surface migration of the deposited atoms due to thermally activated hopping leads to a considerable increase in film density. The bombardment of the film surface by high energy particles induces a significant migration of the deposited atoms and results in a dense structure.
\end{abstract}

Key words: computer simulation, sputter deposition, gas scattering, high-energy particle bombardment

\section{I . INTRODUCTION}

Many researchers have used a Monte Carlo method to investigate sputter deposition processes by computer simulation[1-8]. We have already used that method to investigate the transport and deposition of sputtered particles in a facing target sputtering system and have reported that the selfshadowing effect plays an important role in forming the microstructure of the deposited film [9]. In the present study, we used a computer simulation to clarify the effects of sputterdeposition parameters such as sputtering gas pressure, substrate temperature, and high-energy particle bombardment on the microstructure and composition of the film.

\section{II . SIMULATION MODEL}

Two types of sputtering systems -magnetron sputtering and facing target sputtering (FTS)were simulated (Fig.1). The sputter deposition process was divided into three steps: (1) emission from the target, (2) transport from target to substrate, (3) film formation on substrate. In calculating the emission process, the angular distribution of the emission angles of the sputtered particles was assumed to follow the cosine law. The

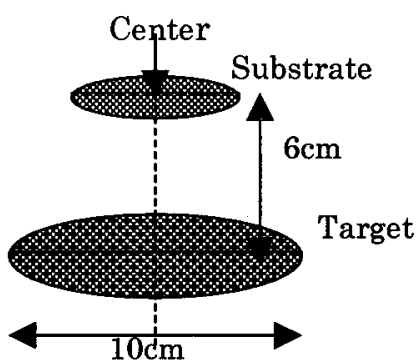

Magnetron sputtering system

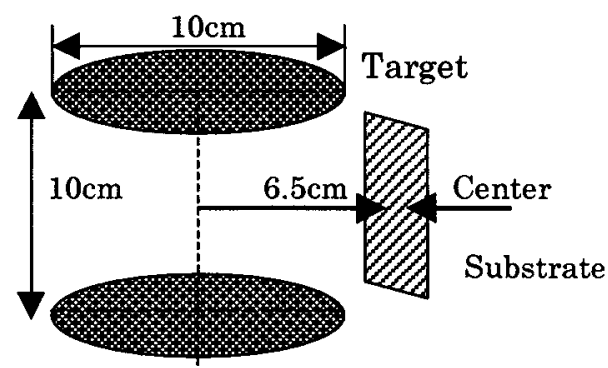

Facing target sputtering system

Fig.1 Sputtering system used in this study.

energy distributions of the sputtered atoms emitted from the target were calculated by using Tompson's model [10].

In calculating the transport process, a model reported by Motohiro [2] and Turner [3] was used to calculate the trajectory of each of the sputtered particles emitted from the target in order to estimate the number of particles deposited on substrate and the distribution of their incident angles to the substrate.

The distribution of incident angles was used in the calculation of the deposition process. The atoms were assumed to be hard spheres and to be deposited on substrate according to a model reported by Henderson et al. [11].

The surface migration of the deposited atoms that was due to thermally activated hopping was introduced by using a model similar those reported by Muller [12] and S. Muller-Pfeiffer et al. [13]. The number $\mathrm{N}$ of hops was calculated as

$N=v_{0} \exp \left(-\frac{\Delta E}{k T}\right)$,

where $\mathrm{k}$ is the Boltzmann constant, $\mathrm{T}$ is temperature, $\Delta \mathrm{E}$ is activation energy, $v_{0}$ is phonon frequency, 
Here

$v_{0}=\left(\frac{8 k T}{\pi m}\right)^{\frac{1}{2}} / d$

where $d$ is interatomic distance, and $\mathrm{m}$ is atomic mass,

The activation energy for the hopping of an atom in contact with three atoms was assumed to be given by

$\Delta \mathrm{E}=\mathrm{Q}=\left(5+\frac{20 \mathrm{~T}}{3 \mathrm{~T}_{\mathrm{M}}}\right) k T_{M}$,

where $Q$ is the activation energy for surface diffusion and $T_{M}$ is melting point.

The activation energy of an atom in contact with more than three atoms was assumed to be given by $\Delta E=(n-3) \phi+Q$,

where $\phi$ is the energy of a single bond.

The effect of the high-energy particle

bombardment on the film surface during deposition was also studied by using a molecular dynamic simulation model reported by Yamamoto et al. [6]

\section{RESULTS AND DISCUSSION}

\section{A. Composition change caused by gas scattering}

Only the scattering of sputtered particles by collision with sputtering gas was considered. Figure 2 shows examples of the simulated changes in the ratio of the amount of iron atoms to barium atoms arriving at the substrate(arrival ratio of iron atom to barium atom) in the deposition of barium ferrite films at various sputtering gas pressure by using FTS. The experimental results were also shown in this figure. It should be noted that the iron content in the film increases as the sputtering gas pressure increases and that it takes a maximum at a certain pressure, above which the iron content decrease. These changes in film composition agree qualitatively with the experimental results. Figure 3 shows, for magnetron sputtering, the changes in the arrival ratio of iron atom to barium atom with changes in sputtering gas pressure. The iron content in the film decreases monotonically as the sputtering gas pressure increases, which is quite different from that observed in FTS, shown in Fig. 2. These results suggest that the scattering of the sputtered particles by the collision with sputtering gas should be taken into consideration if we want to control the film composition.

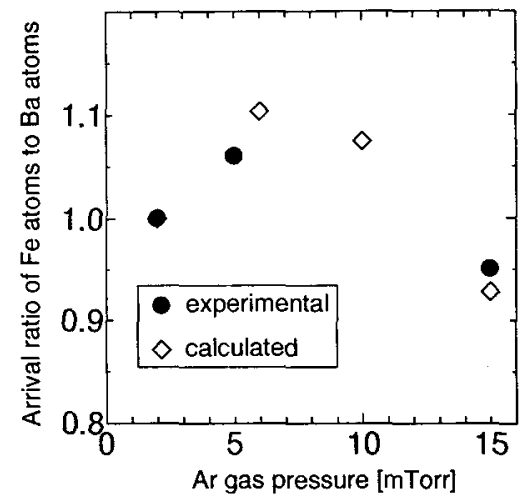

Fig.2 Changes in the arrival ratio of iron atoms to barium atoms with Ar gas pressure in the deposition of barium ferrite films by using FTS.

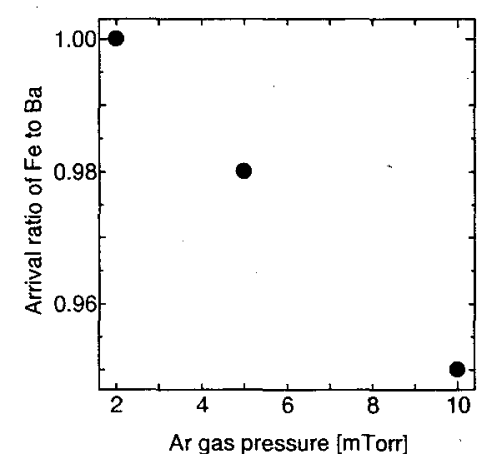

Fig.3 Changes in the arrival ratio of iron atoms to barium atoms with changes in Ar gas pressure (magnetron sputtering).

\section{B. Surface migration of deposited atoms due to thermally activated hopping}

Figure 4 shows the examples of the microstructure simulated for cobalt films deposited when the FTS system is used at 2 mTorr and at 10 $\mathrm{m}$ Torr without surface migration. It is clear that the film deposited at a higher gas pressure has a lower density. This is a result of the self shadowing effect being more prominent at higher pressures.

The effect of surface migration of the deposited atoms on the microstructure of the film due to thermally activated hopping was investigated by depositing the film at various temperatures. Figure 5 shows a simulated microstructure of cobalt films deposited, using the FTS system at $500 \mathrm{~K}$, and $600 \mathrm{~K}$. The argon gas pressure and deposition rate were fixed at 10 mTorr, and $100 \AA / \mathrm{min}$, respectively. Comparing the structure of this film with that of the film deposited without surface migration, shown in Fig. 4(b), we can easily see that both film density and grain size are increased significantly by surface migration of the deposited atoms and surface roughness decreases remarkably. These results indicate that the control of the distribution of the 

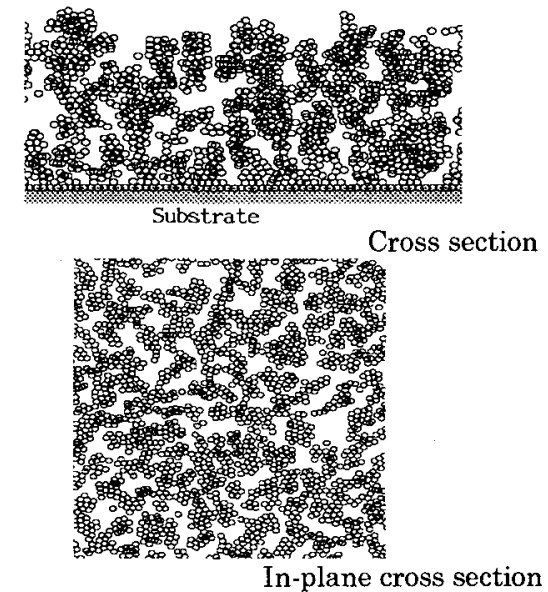

(a) Ar gas pressure : 2 mTorr

In-plane cross section

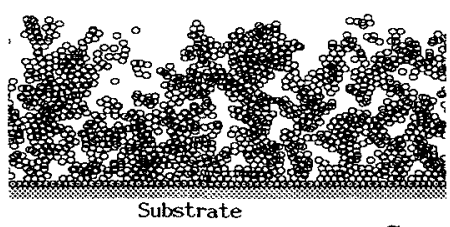

Cross section

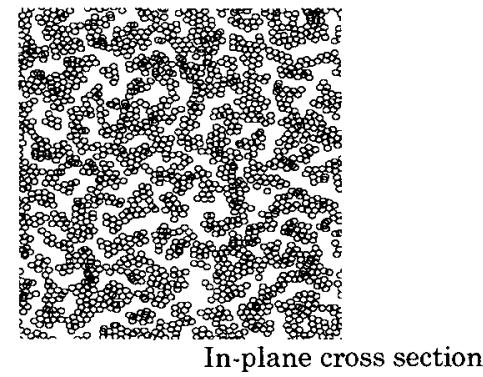

(b) Ar gas pressure : 10 mTorr

Fig. 4. Simulated microstructure of the cobalt films deposited using the FTS, at (a) 2 mTorr and (b) 10 mTorr.

incidence angles of the deposition particles on the substrate is important when the film is deposited at a low temperature where surface migration is suppressed to obtain a film with desired microstructure.

\section{High energy particle bombardment of film surface}

The effect of the high-energy particles bombarding the film surface during deposition was also studied by using a molecular dynamic simulation. Argon ions with various energies bombarded the surface of the film deposited at 500 $\mathrm{K}$, and $10 \mathrm{~m}$ Torr by using FTS. Figure 6 shows examples of simulated movement of the atoms in the film induced by the bombardment of a highenergy argon ion. The bombardment of argon ions with an energy of $20 \mathrm{eV}$ can induce the migration

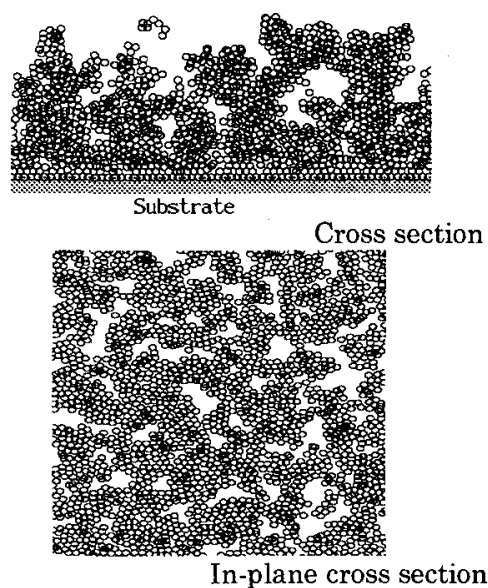

(a) Deposition temperature : $500 \mathrm{~K}$
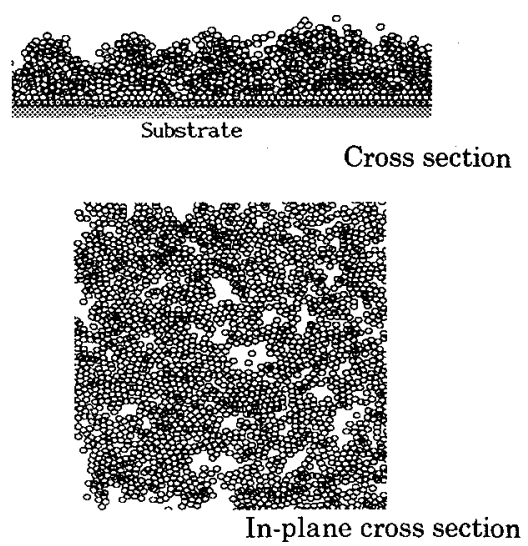

(b) Deposition temperature : $600 \mathrm{~K}$

Fig. 5. Simulated microstructure of cobalt films deposited using the FTS at (a) $500 \mathrm{~K}$ and (b) $600 \mathrm{~K}$. (Ar gas pressure : $10 \mathrm{mTorr}$, deposition rate $: 100 \dot{A} / \mathrm{min}$ )

of the atoms in the film. This indicates that the high-energy particle bombardment can induce a significant migration of the deposited atoms in the film and results in a densification of the film. Figure 7 shows the changes in the simulated microstructure of the films caused by the bombardment of argon ions with an energy of 50 $\mathrm{eV}$. It is evident from the figure that the bombardment causes the film density to increase.

\section{CONCLUSIONS}

This computer simulation of sputter deposition processes yielded the following results: (1) The composition of a film with elements of different masses changes with sputtering gas pressure as a result of the sputtered particles scattering when they collide with atoms of the 


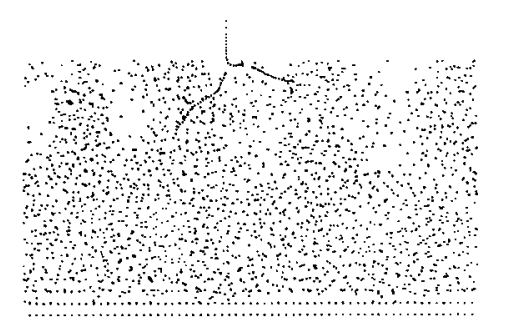

(a) Bombardment of Ar ion with $20 \mathrm{eV}$

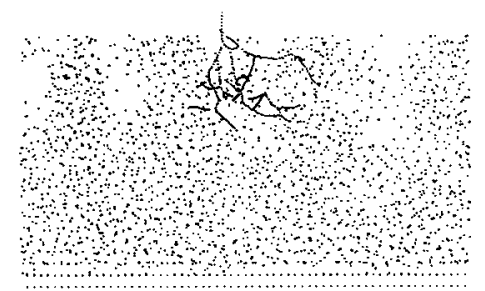

(b) Bombardment of $\mathrm{Ar}$ ion with $50 \mathrm{eV}$

Fig. 6 Simulated movement of atoms in the film caused by a bombardment of a high energy argon ion.

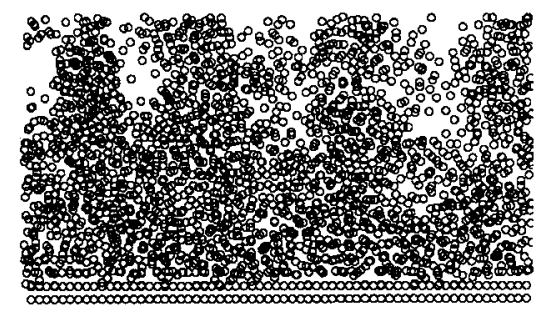

(a) Simulated microstructure of the film before Ar ion bombardment.

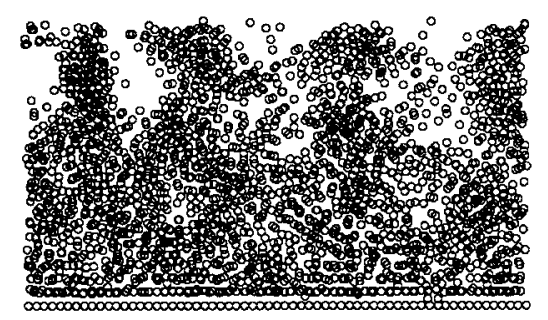

(b) Simulated microstructure of the film after Ar ion bombardment.(10 shots of ions with $50 \mathrm{eV}$ ).

Fig. 7 Changes in simulated microstructure of the films induced by the bombardment of argon ions.

sputtering gas, and it became clear that the gas pressure dependence of the film composition in magnetron sputtering differs clearly from that in facing target sputtering. (2) The density of a film is increased significantly by the surface migration of deposited atoms. (3) The bombardment of the film by high energy particles induces a significant migration of the deposited atoms and results in a dense structure.

\section{REFERENCES}

[1] J.P. Bieersack and W. Eckstein, "Sputtering studies with the Monte Carlo program TRIM.SP," Appl. Phys., A34, 73 (1984).

[2] T. Motohiro, "Applications of Monte Carlo simulation in the analysis of a sputter-deposition process,” J. Vac.. Sci. Technol., 4 (2), 189 (1986).

[3] G.M. Turner, I.S. Falconer,B.W. James, and D.R. Mckenzie, "Monte Carlo calculation of the thermalization of atoms sputtered from the cathode of a sputtering discharge," J. Appl. Phys., 65 (9), 3671 (1989)

[4] A.M. Myers, J.R. Doile, J.R. Abelson, and D.N. Ruzic, "Monte Carlo simulation of magnetron sputtering particle transport," J. Vac. Sci. Technol., A9(3) ,614(1991).

[5] H. Yamada,T. Shinmura, Y. Yamada, and T. Ohta, "Practical Monte Carlo sputter deposition simulation with quasi-axis-symmetrical(QAS) approximation," IEEE IEDM94, 553 (1994).

[6] A.G. Dirks, and H.J. Leamy, "Columnar microstructure in vapor-deposited thin films," Thin Solid Films, 47,219 (1977).

[7] S. Valkealahti and R.M. Nieminen,, "Molecular dynamic simulation of the damage production in $\mathrm{Al}(11)$ surface with slow argon ions," Nucl. Instr. and Meth. B18,365-369 (1987).

[8] H. Yamada, T. Shinmura, and T. Ohta, Sputter target ejection angle distribution simulation with molecular dynamics," Technical Report of IEICE Japan, VLD95-53 (1995) in Japanese.

[9] Y. Hoshi and E. Suzuki, "Changes in angular distribution of incident sputtered particles in sputter deposition of iron films," J. Mag. Soc. Jpn, 18, S1, 323-326 (1994).

[10] M.W. Thompson, "The energy spectrum of ejected atoms during the high energy sputtering of gold," Phylis. Mag., 18, 377-414 (1969).

[11] D. Henderson, M.H. Brodsky, and P. Chaudari, "Simulation of structural anisotropy and void formation in amorphous thin films," Appl. Phys. Lett., 25 (11) 641 (1974).

[12] Karl-Heinz Muller, "Dependence of thin film microstructure on deposition rate by means of a computer simulation," J. Appl. Phys., 58 (7) 2573 (1985).

[13] S. Muller-Pfeiffer, H.van Kranenburg, and J.C. Lodder, "A two-dimensional Monte Carlo model for thin film growth by oblique evaporation: simulation of two-component systems for example of CoCr," Thin Solid Films, 213, 143-153 (1992). 